\title{
ANFIS Tuned No-Reference Quality Prediction of Distorted/Decompressed Images featuring Wavelet Entropy
}

\author{
Indrajit De ${ }^{1}$, Jaya Sil ${ }^{2}$ \\ ${ }^{1}$ Department of Information Technology \\ MCKV Institute of Engineering \\ Howrah, West Bengal, India \\ Indrajitde2003@yahoo.co.in \\ ${ }^{2}$ Department of Computer Science and Engineering \\ Bengal Engineering and Science University, Shibpur \\ Howrah, West Bengal, India \\ js1@cs.becs.ac.in
}

\begin{abstract}
Assessing quality of distorted/decompressed images without reference to the original image is difficult due to vagueness in extracted features and complex relation between features and visual quality of images. The paper aims at assessing the quality of distorted/decompressed images without any reference to the original image by developing an adaptive network based fuzzy inference system (ANFIS). First level Haar approximation entropies of test images from LIVE database and region based features extracted from the benchmark images are considered as inputs while mean opinion score (MOS) based quality of the images used as output to the fuzzy inference system (FIS). The input-output variables of the FIS are expressed using linguistic variables and fuzzified to measure the vagueness in extracted features. Takagi-Sugeno-Kang (TSK) inference rule has been applied to the FIS to predict the quality of a new distorted/decompressed image. The FIS has been trained to tune the parameters of the membership functions of the fuzzy sets that assess quality of the image more accurately. Quality of decompressed and various noise incorporated distorted test images are predicted using the proposed method producing output comparable with other existing no reference techniques. Results are validated with the objective and subjective image quality measures.
\end{abstract}

Keywords: Fuzzy Systems, Gaussian Noise, Image Compression, MOS, Wavelet entropy, ANFIS.

\section{Introduction}

Digital images are subjected to loss of information, various ways of distortions during compression and transmission, which deteriorate visual quality of the images at the receiving end. Quality prediction of an image is therefore, important. Modeling physiological and psycho visual features of the human visual system and signal fidelity criteria based quality assessment are reported $[10,11]$ though each of these approaches has several shortcomings. Since human beings are the ultimate consumers of almost all the image content, the most reliable means of measuring the image quality is subjective evaluation based on the opinion of the human observers [7, 14]. However, subjective testing is not automatic and expensive too. On the other hand, most objective image quality assessment methods [5,8] either require access to the original image as reference [2] or only can evaluate images, degraded with predefined distortions and therefore, lacking generalization approach. One work [3] has been reported very recently, which needs partial information in the form of a set of extracted features rather than the full access to the reference image. However, in this approach extra cost is involved to transmitting additional information along with the compressed image to the other end. Two prominent works have been reported relating to no-reference image quality evaluation, (i) Wang, Bovic and Shiekh's no-reference JPEG image quality index and (ii) H.Shiekh's quality metric based on natural scene statistics (NSS) model applied on JPEG2000 compressed images. Wang et al's work is computationally inefficient since quality of image is predicted after combining the features extracted by scanning the image both horizontally as well as vertically.

\section{A. Review Work}

The work of Wang et. al. considers blocking and blurring as the major features of a JPEG compressed image, derived during quantization step of the compression process. To obtain the features, first the image is transformed into frequency domain and differencing signal $d_{h}(m, n)$ is calculated along each horizontal line of the image, using equation (1). The blocking effect is estimated as the average difference across the block boundaries. Blurring causes reduction of signal activity, evaluation of which gives more insight of the relative blur in the image.

$$
d_{k}(m, n)=x(m, n+1)-x(m, n)
$$

where the test image signal is $x(m, n)$ and $m \in[1, M], n \in[1$, $N]$. 
Secondly, to measure the blurring feature the activity of the image signal is estimated because blurring is difficult to be evaluated without the reference image. The activity of the image signal is measured using two factors. The first one is the average absolute difference between in-block image samples while the second one is the zero-crossing (ZC) rate. All such features are calculated along horizontal and vertical direction and then combined to get the resultant features. The horizontal blocking feature $B_{h}$ is derived using (2).

$$
B_{h}=\frac{1}{M(\lfloor N / 8\rfloor-1)} \sum_{i=1}^{M} \sum^{\lfloor N / 8\rfloor-1}\left|d_{h}(i, 8 j)\right|
$$

The blurring effect of an image sample is measured using (3).

$$
A_{h}=\frac{1}{7}\left[\frac{8}{M(N-1)} \sum_{i=1}^{M} \sum_{j=1}^{N-1}\left|d_{h}(i, j)\right|-B_{h}\right]
$$

The second activity measure $Z_{h}$ is the horizontal $\mathrm{ZC}$ rate can be estimated by (4).

$$
Z_{h}=\frac{1}{M(N-2)} \sum_{i=1}^{M} \sum_{j=1}^{N-2} z_{h}(m, n)
$$

where $n \in[1, N-2]$ and at $d_{h}(m, n), z_{h}(m, n)=1$ which is horizontal $\mathrm{ZC}$.

Using similar methods, the vertical features $B_{v}, A_{v}$, and $Z_{v}$ are computed. Finally, the respective image features $B, A$ and $Z$ are obtained as summarized in (5).

$$
B=\frac{B_{h}+B_{v}}{2}, A=\frac{A_{h}+A_{v}}{2}, Z=\frac{Z_{h}+Z_{v}}{2}
$$

The quality of the image is now calculated, using (6)

$$
S=\alpha+\beta B^{\gamma 1} A^{\gamma 2} Z^{\gamma 3}
$$

where $\alpha, \beta, \gamma 1, \gamma 2$ and $\gamma 3$ are the model parameters, estimated using subjective test data by applying nonlinear regression technique.

There are several drawbacks to this method. First, the process has taken only JPEG compressed images as its reference. Secondly, for training subjective data has been used in the form of mean opinion score and lastly local spatial regional texture variations of the concerned image are not considered to design the quality metric.

The work of H. Sheikh et.al assesses the quality of images affected with ringing and blurring distortion resulting from JPEG2000 compression. In their approach natural scene statistics (NSS) models are applied to provide a 'reference' against which the distorted images can be assessed. Here, subbands of a JPEG2000 compressed image are considered. Sinceas difference exists among the features of the subbands, the work proposes independent nonlinear transform of each subband feature before combining them linearly. The nonlinear transformation is designed to improve correspondence among the features of different subbands. Six subbands of the image are considered: horizontal, vertical and diagonal orientations at the second-finest and at the finest resolution. The transformations are obtained using training data and by fitting image quality to the features from individual subband, described in (7).

$$
q_{i}=K_{i}\left(1-\exp \left(-\left(p_{s s, i}-u_{i}\right) / T_{i}\right)\right)
$$

where $q_{i}$ is the transformed feature (predicted image quality) for the $i$-th subband, $p_{s s, i}$ is the probability for the $i$-th subband, and $K_{i}, T_{i}$ and $u_{i}$ are curve fitting parameters for the $i$-th subband that are learned from the training data. Since each subband feature is mapped to subjective quality by nonlinear transformation, the features from all subbands are approximately aligned with subjective quality, and hence with each other as well. A weighted average of the transformed feature is used for quality prediction. Due to the similarity in the statistics of horizontal and vertical subbands at a particular scale, they constrain the weights to be the same for these orientations at a given scale. Thus, the six-dimensional subband quality vector, $q=\{q i \mid i \in 1 . .6\}$, is modified into a four-dimensional vector $q l$ by averaging the predicted quality from horizontal and vertical subbands at a given scale. The final quality prediction is taken to be a weighted average of $q l$ defined by (8).

$$
\begin{aligned}
& q l:\left[\begin{array}{c}
q l 1 \\
q l 2 \\
q l 3 \\
q l 4
\end{array}\right]=\left[\begin{array}{c}
(q 1+q 2) / 2 \\
q 3 \\
(q 4+q 5) / 2 \\
q 6
\end{array}\right] \\
& Q=q l^{T} w
\end{aligned}
$$

$Q=$ predicted quality value of the image and the weights $w$ are learned by minimizing quality prediction error over the training set. $P$ ss, the probability that the wavelet predictor/coefficient pair obtained for a given subband of natural scene images are significant. Like the previous work, the model does not consider any spatial texture variation of the image due to which local statistics of the image may vary. Moreover, the work is highly influenced by the nature and number of statistical parameters.

To address the shortcomings of the existing methods, both subjective and objective measures of image information are utilized in framing the rule base of the FIS. Five benchmark gray level decompressed images (Lena, Mandril, Woman with Hat, Woman_Blond_Hair and Baby) are divided into different regions and crisp value of input features such as area, extent and eccentricity of these regions are obtained and represented using linguistic variables. Similarly, as output of the system, quality of the decompressed image is measured using MOS and thus, subjective judgment has been 
incorporated. First level Haar approximation entropies of test images from LIVE database and region based features are measured to quantify the information by objective means. In consultation with human observers various fuzzy if-then rules are constructed using input-output features and finally, by applying TSK inference rule [1], the quality of a new distorted/decompressed image is predicted without any reference to the original image. ANFIS [18] has been implemented to tune the parameters of membership functions of fuzzy sets, which were prefixed based on experts' opinions and therefore, subjective and uncertain. Results are compared with the existing no-reference image quality metrics and validated with peak signal to noise ratio (PSNR) and mean opinion score (MOS), which are standard objective and subjective image quality measures.

The paper is divided into five sections. Section II describes the model while the proposed method of image quality assessment is presented in section III. Results are demonstrated in section IV and finally conclusions summarized in section $\mathrm{V}$.

\section{The Model}

\section{A. The TSK model}

The proposed model consists of five components:

(a) Input-Output space: In the problem domain the input space is comprised of first level Haar approximation entropies along with three parameters, area, extent and eccentricity. Similarly, quality of image measured using MOS are mapped as output of the FIS, given in TABLE I.

TABLE I. QUALITY OF IMAGES

\begin{tabular}{|l|l|l|}
\hline MOS & Quality & Impairment \\
\hline 10 & Good & Imperceptible \\
\hline 6 & Average & $\begin{array}{l}\text { Perceptible but not } \\
\text { annoying }\end{array}$ \\
\hline 3 & Bad & Slightly annoying \\
\hline 1 & Poor & Annoying \\
\hline
\end{tabular}

(b) In order to accommodate human's observations and expressions crisp value of the input-output parameters are mapped into different linguistic term sets. A fuzzy rule base is built using the linguistic termsets representing the relationship between the physiological impression of humans and quality of of the image. Thus in the rule base reasoning power of human being is incorporated. The general form of the rules in multi-input-single-output (MISO) FIS is:

If 'Area_Values' are 'Good' ( 0 pixel per region to 3 pixel per region) and 'Extent_Values' are 'Good' (0.5 to 1) and 'Eccentricity_Values' are 'Good' (0 to 0.5$)$ and 'Wavelet Entropy_Values' are 'Low' (3-4) then 'quality' is 'Good' $(0 . \overline{7}$ to 1$)$.

(c) Fuzzification: The crisp measured data of different input-output variables are fuzzified using Gaussian membership function with varied mean and standard deviation, shown in Fig 1. For instance, measured area values are partitioned into three fuzzy sets- good, average and bad with the help of the following functional relation:

$f($ area $)=\operatorname{good}(0<=$ area $<=4)$ or average $(5<=$ area $<=7)$ or bad $(8<=$ area $<=10)$

After fuzzification the area value equal to 7 is classified as $0.2 \%$ of average and $0.8 \%$ of bad.

(d) Inference engine: The max-min composition operator and min operation of fuzzy implication rule is used for computational simplicity and efficiency to generating the inferences.

(e) Defuzzifier: The weighted average method [1] is used for defuzzification of output fuzzy variables.

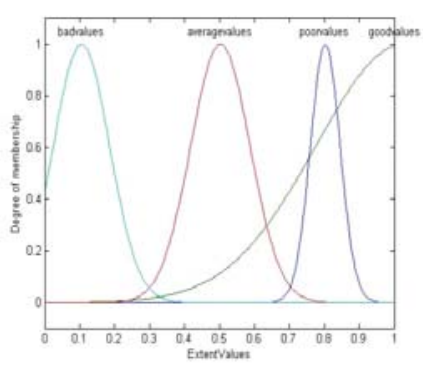

(a)

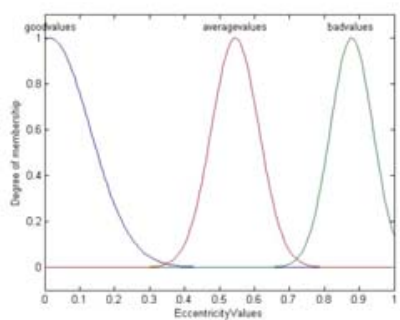

(b)

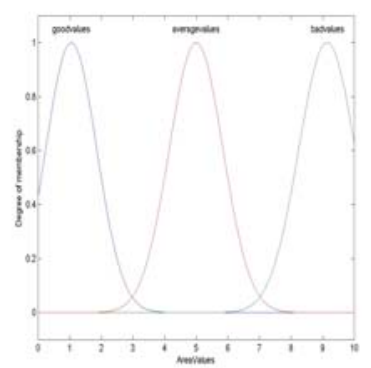

(c)

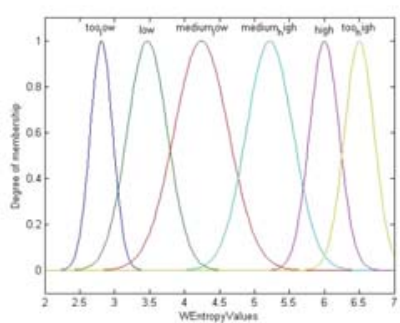

(d)

Figure 1. Membership functions: (a) Extent, (b) Eccenricity, (c) Area, (d) Wavelet Entropy 
TABLE II. RANGE OF VALUES OF DIFFERENT FUZZY VARIABLES

\begin{tabular}{|c|c|c|c|c|}
\hline $\begin{array}{l}\text { Area (pixels } \\
\text { per region) }\end{array}$ & Extent & $\begin{array}{l}\text { Eccentricit } \\
\mathrm{y}\end{array}$ & $\begin{array}{l}\text { Wavelet } \\
\text { entropy value }\end{array}$ & Quality \\
\hline $\begin{array}{l}\text { Good (1 to } \\
3)\end{array}$ & $\begin{array}{l}\text { Good } \\
(0.4 \text { to } \\
1.0) \\
\end{array}$ & $\begin{array}{l}\text { Good (0 to } \\
0.3)\end{array}$ & $\begin{array}{l}\text { Medium } \\
\operatorname{low}(3.5-5.0)\end{array}$ & $\begin{array}{l}\text { Good }(0.6 \text { to } \\
1)\end{array}$ \\
\hline $\begin{array}{l}\text { Good (1 to } \\
3 \text { ) }\end{array}$ & $\begin{array}{l}\text { Good } \\
(0.4 \text { to } \\
1.0) \\
\end{array}$ & $\begin{array}{l}\text { Good (0 to } \\
0.3)\end{array}$ & $\operatorname{low}(3-4)$ & $\begin{array}{l}\text { Good }(0.7 \text { to } \\
1)\end{array}$ \\
\hline $\begin{array}{l}\text { Average (4 } \\
\text { to } 6)\end{array}$ & $\begin{array}{l}\text { Avera } \\
\text { ge }(0.4 \\
\text { to } 0.6) \\
\end{array}$ & $\begin{array}{l}\text { Average } \\
(0.4 \text { to } 0.6)\end{array}$ & $\operatorname{High}(5.5-6.5)$ & $\begin{array}{l}\text { Average }(0.4 \\
\text { to } 0.6)\end{array}$ \\
\hline $\begin{array}{l}\text { Average (4 } \\
\text { to } 6)\end{array}$ & $\begin{array}{l}\text { Avera } \\
\text { ge }(0.4 \\
\text { to } 0.6) \\
\end{array}$ & $\begin{array}{l}\text { Average } \\
(0.4 \text { to } 0.6)\end{array}$ & $\begin{array}{l}\text { medium } \\
\text { high(4.5-6) }\end{array}$ & $\begin{array}{l}\text { Average }(0.4 \\
\text { to } 0.6)\end{array}$ \\
\hline Bad (7 to 10$)$ & $\begin{array}{l}\text { Bad }(0 \\
\text { to } 0.3) \\
\end{array}$ & $\begin{array}{l}\operatorname{Bad}(0.7 \text { to } \\
1.0)\end{array}$ & Too high(6-7) & poor $(0$ to 0.2$)$ \\
\hline $\operatorname{Bad}(7$ to 10$)$ & $\begin{array}{l}\text { Poor } \\
(0.7 \text { to } \\
0.9)\end{array}$ & $\begin{array}{l}\operatorname{Bad}(0.7 \text { to } \\
1.0)\end{array}$ & $\begin{array}{l}\text { Too } \\
\operatorname{low}(2.5-3.5)\end{array}$ & $\begin{array}{l}\text { bad }(0.2 \text { to } \\
0.3)\end{array}$ \\
\hline
\end{tabular}

\section{B. Designing of Membership functions}

The range of membership values of input fuzzy variables (good, bad, average and poor) are determined with respect to the number of regions (NOR). Frequency distributions of three input parameters for different training images are obtained and partitioned to different ranges corresponding to linguistic terms in order to frame the fuzzy rules of the proposed FIS as shown in Fig 2.

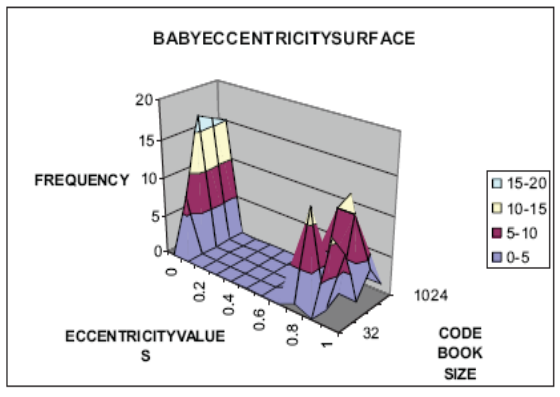

(a)

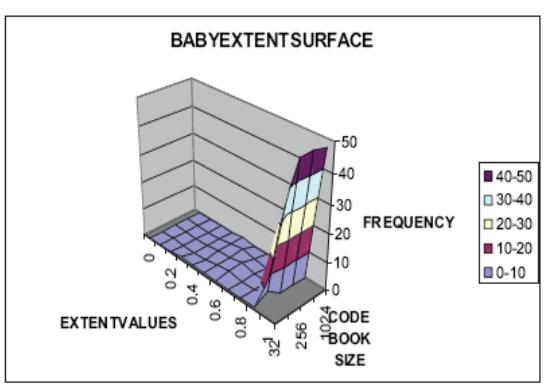

(b)

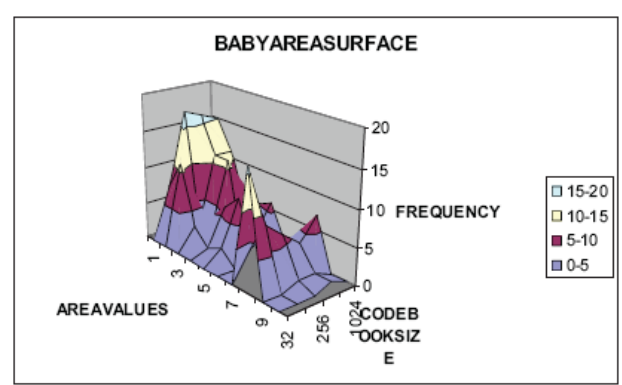

(c)

Figure 2. Frequency distributions of regional feature( Area, Extent and Eccentricity) values with respect to codebook size for the training image Baby

In general, maximum frequencies of individual input parameters are considered to calculate the quality value of images using the proposed FIS. However, if input parameters of images are appeared with equal maximum frequencies then weighted average value of individual parameters are taken to calculate the quality of a decompressed image. In building the FIS, the shape of the membership functions are decided from the shape of the frequency distribution curve and the range is decided through human visual perception and therefore, needs fine tuning to predict the quality of images more accurately. The distribution of wavelet entropies with respect to NOR is also prepared and applying similar reasoning the fuzzy variables are designated (too low, low, medium_low, medium_high, high, too_high). Similarly, human observers are involved to fixing the range of membership value of quality of images based on MOS (TABLE I). TABLE II summarizes the mapping of fuzzy variable to membership value for all input-output features.

\section{Image Features}

Area is defined as the number of pixels in a particular region and this number is inversely proportional to granularity of the image. For good quality images, resolution is better indicates increasing value of NOR. Therefore, number of pixels per region becomes less and in extreme case it becomes one per region since total number of pixels remaining constant. For bad quality images the reverse happens. Figure. 3 illustrates that the quality of an image is described using PSNR with respect to the original image, which ascertain that the relation between PSNR and NOR generally linear. The first level Haar approximation entropies of the test images of LIVE database [5] with respect to NOR are plotted in Fig. 4. It has been observed that the wavelet entropy value is in general, inversely proportional to NOR and it is quite obvious since NOR actually represent the total number of connected components present in an image. So if there is initially " $n$ " number of components then the probability of occurrence of an individual component is $1 / n$. With increasing number of connections amongst the components, ' $n$ ' decreases and the probability increases, since the wavelet entropy is expressed as $-\sum p \log p$, where $p=$ probability of occurrence of concerned wavelet coefficient. The spatial frequency ' $f$ ' representing the wavelet coefficient component of a level $\lambda$ is directly proportional to display resolution $r$ pixels/degree and inversely proportional to $2^{\lambda}[12]$. So for the same level, the pixel component and the wavelet coefficient component are directly related and for both, the variation produces equivalent 
effect. Therefore, with the increase in the probability of occurrence of connected components (essentially connected pixel components), the wavelet coefficient probability increases giving rise to decrease in entropy. Area and Wavelet_entropy (WE) values decrease with increase of NOR. Fuzzy C means clustering algorithm [13], has been applied on Area and Wavelet Entropy data in order to group the data corresponding to their degree of membership value. Fuzzy c-means (FCM) clustering processes $n$ vectors in $p$-space as data input, and uses them, in conjunction with first order necessary conditions for minimizing the FCM objective function, to obtain estimates for two sets of unknowns. The cluster centers are shown in Fig. 5, which establishes the fact that nearer the "Area-value" $=1$ the wavelet entropies get minimized, thereby reducing the randomness of the system.

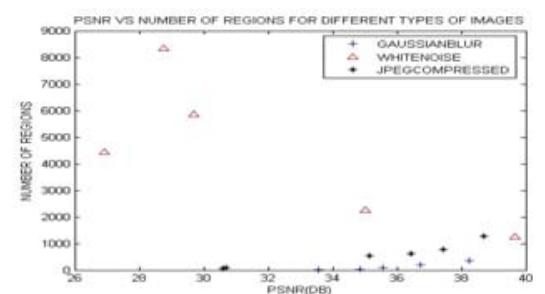

Figure 3. PSNR vs Number of regions.

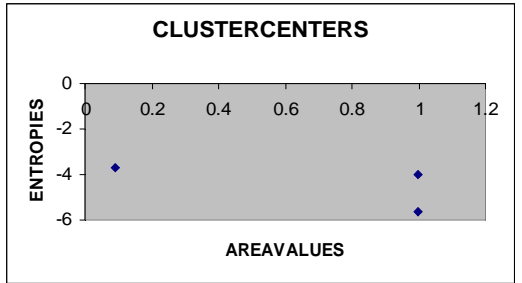

Figure 5. Area Values vs Wavelet Entropy

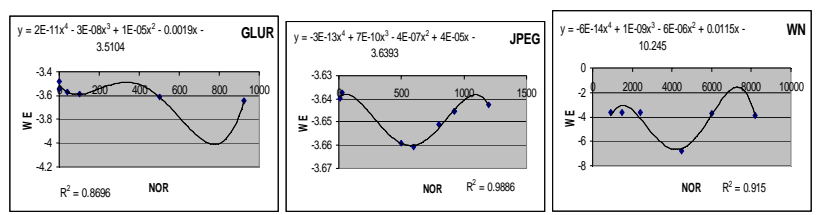

Figure 4. Wavelet Entropy vs NOR of Test Images(a) Gaussian Blur (b) Jpeg (c) White noise

Extent is the proportion of the pixels in the rectangle, surrounding the concerned region. Selection of extent follows similar justifications as area. The eccentricity of the ellipse has the same second-moments as the region concerned. For good quality images having large number of regions, the ellipse concerned almost encompasses one pixel per region resembling a circle and therefore, eccentricity tends to zero for them. Other values nearer to one occur for average to bad quality images.

\section{Procedure}

Five standard gray level benchmark images- Lena, Mandril, Woman with Hat, Woman_Blond_Hair and Baby (Figure 6) are used to generalizing the fuzzy rules of the proposed FIS. The images are converted to gray level images and then to

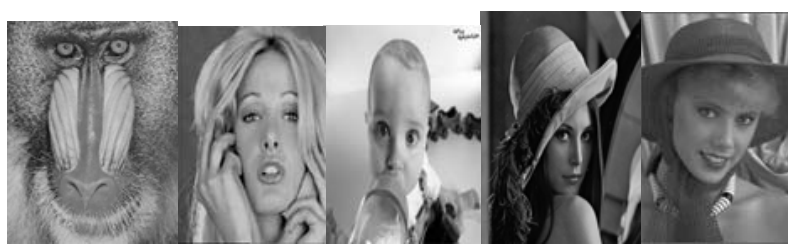

(a)

(b)

(c)

(d)

(e)

Figure 6. Training images (a) Mandril, (b) Woman_blond_hair, (c) Baby, (d) Lena, (e) woman_with_hat

JPEG images so that our method can be compared with other existing methods $[3,4]$, which are applicable only for JPEG images. The images are decompressed using Linde Beuzo Gray (LBG) algorithm [6] with codebooks of different sizes like 32, 128, 256, 512 and 1024 and from each decompressed images, area, extent, eccentricity and quality values are obtained.

TABLE IV. COMPARISONS OF QUALITY METRICS

\begin{tabular}{|c|c|c|}
\hline Image name & $\begin{array}{l}\text { Proposed quality- wavelet } \\
\text { entropy based(linguistic } \\
\text { expression)/MOS/PSNR }\end{array}$ & $\begin{array}{l}\text { Proposed quality (linguistic } \\
\text { expression) /MOS/PSNR }\end{array}$ \\
\hline \multicolumn{3}{|c|}{ Gaussian blur } \\
\hline img132 & 6 (average) / 8.28 / 32.63 & 5.5 (average) / $8.28 / 32.63$ \\
\hline img162 & $\begin{array}{l}9 \text { (good) / 5.43 / Equivalent } \\
\text { to original }\end{array}$ & $\begin{array}{l}9.01 \text { (good) / } 5.43 / \\
\text { Equivalent to original }\end{array}$ \\
\hline img36 & 8.49 (good) / $10 / 34.07$ & $3.3(\mathrm{bad}) / 10 / 34.07$ \\
\hline img42 & 8.72 (good) / $10 / 34.79$ & 8.72 (good) / $10 / 34.79$ \\
\hline img61 & 9.01 (good) / $10 / 36$ & 9.01 (good) / $10 / 36$ \\
\hline \multicolumn{3}{|l|}{\begin{tabular}{|l|} 
Jpeg \\
compressed \\
\end{tabular}} \\
\hline img138 & $3.16(\mathrm{bad}) / 1.09 / 30.69$ & $3.3(\mathrm{bad}) / 1.09 / 30.69$ \\
\hline img1 & 6 (average) / $7.57 / 35.09$ & 8.95 (good) / $7.57 / 35.09$ \\
\hline img107 & 6.01 (average) / $7.71 / 37.35$ & 9.01 (good) / 7.71/37.35 \\
\hline img154 & 6 (average) / $1.09 / 30.76$ & 5.5 (average) / $1.09 / 30.76$ \\
\hline img168 & 9 (good) / $4.19 / 38.63$ & 9.01 (good) / $4.19 / 38.63$ \\
\hline \multicolumn{3}{|l|}{ white noise } \\
\hline img11 & 9 (good) / 5.14 / 39.52 & $9.01($ good) / $5.14 / 39.52$ \\
\hline img119 & 8.9 (good) $/ 1.47 / 28.90$ & $9.01($ good $) / 1.47 / 28.90$ \\
\hline img162 & $\begin{array}{l}9 \text { (good) / } 6.42 \text { / equivalent to } \\
\text { original }\end{array}$ & $\begin{array}{l}9.01 \text { (good) / } 6.42 / \text { equivalent } \\
\text { to original }\end{array}$ \\
\hline img28 & 9 (good) / $1.95 / 29.72$ & $9.01($ good) / $1.95 / 29.72$ \\
\hline img60 & 9 (good) / $1 / 28.23$ & 9.00 (good) / $1 / 28.23$ \\
\hline
\end{tabular}

\section{A. The Rule Base}

Simultaneously genetic algorithm [17] is applied to optimize the objective function representing the distribution of first level Haar approximation entropies with respect to NOR.

Then the optimized entropy values are partitioned by correlating their correspondences with the MOS of the considered images to frame the rule base of the FIS along with the other input-output features. After building of FIS, standard test 'face' images have been considered from the LIVE database of H.R.Sheikh-release-2 [5]. In the database, images are available with several types of distortions, out of 
which three categories have been taken for testing: Gaussian blur, JPEG compressed and white noise incorporated images (see, Fig. 8). The input and output features of these test images are extracted and applied to the FIS for assessing quality of the images.

TABLE III. RESULTS OF ANFIS (BACKPROPAGATION METHOD)

\begin{tabular}{|l|l|l|l|}
\hline EPOCH & $\begin{array}{l}\text { ERROR } \\
\text { TOLERANC } \\
\text { E }\end{array}$ & ATRE & ATSE \\
\hline 3 & 0.3 & 0.60 & 0.484 \\
\hline 3 & 0.6 & 0.481 & 0.597 \\
\hline 3 & 0.7 & 0.479 & 0.595 \\
\hline 6 & 0.7 & 0.477 & 0.591 \\
\hline 10 & 0.7 & 0.478 & 0.588 \\
\hline 10 & 0.8 & 0.472 & 0.584 \\
\hline 10 & 0.5 & 0.452 & 0.540 \\
\hline 10 & 1.0 & 0.449 & 0.536 \\
\hline 10 & 2.3 & 0.472 & 0.531 \\
\hline 10 & 8.0 & 0.445 & 0.527 \\
\hline 10 & 10 & 0.472 & 0.527 \\
\hline 60 & 1 & 0.439 & 0.514 \\
\hline 100 & 10 & 0.441 & 0.519 \\
\hline 500 & 1 & 0.438 & 0.510 \\
\hline
\end{tabular}

\section{B. ANFIS}

In building the FIS, the range of membership functions are decided through human visual perception and therefore, needs fine tuning to predict the quality of images more accurately. Three different data sets have been prepared; training data set, testing data set and verification data set. The verification data set prevents over fitting of training process to building the ANFIS. During training the root-mean-square error (RMSE), known as average testing error (ATSE) is calculated by taking the difference between the outputs generated by the FIS while applying in sequence, the testing and the training data set as input. Similarly, the RMSE known as average training error (ATRE) computed taking the difference between the outputs of the FIS and the ANFIS when the training data set is fed as input for each of the cases. ATSE and ATRE are listed in TABLE III for any combination of training, testing and verification data set, with pre-assigned number of epochs and the maximum permissible RMSE (error tolerance). For large number of passes (epochs) the two errors converge to nearly equal values, proving robustness of the proposed system. Meanwhile for all the testing images, PSNR and MOS based on the opinion of 25 observers are computed for subsequent validation. The proposed method is applied on different noise incorporated face images exhibiting similar results

\section{Results and Discussions}

\section{A. Comparisons on quality metrics}

In TABLE IV, quality metrics of test images obtained using the proposed and other two methods [14] are compared. From TABLE IV, it is evident that the proposed method generates almost same inferences that of Wang et. al. whereas the process of Shiekh et. al. produced hayware results with respect to images, namely 'img36' and 'img 154'. Wang et. al. process gave wrong result for certain images (img138, img154) while the proposed method yielded coherent results with respect to subjective (MOS) and objective quality (PSNR) judgment of images. In TABLE V, quality metric obtained using the proposed FIS are shown with and without using wavelet entropy, which more closely resembles the subjective judgment of the images.

\section{B. Validation of result}

Figure 7 describes comparison of MOS with PSNR for FIS based quality metric of different distorted images. From the figure it is evident that both the proposed FIS based quality metric and MOS performances are equivalent with respect to the distorted images.

\section{Conclusions}

Pearson product moment correlation coefficient (PPMCC) is a powerful measure to establish linearity between two variables. The PPMCC has been calculated to measure the performance of the proposed method and the other existing methods

in comparison with PSNR, in TABLE V. In TABLE VII, to show prediction monotonicity, Spearman rank order correlation coefficient [15]

has been computed between PSNR and different image quality metrics. PPMCC has been calculated for different noise incorporated images too, which exhibits the proposed process outperforms H.Sheikh, et. al.'s procedure and almost at per with Wang et. al. Moreover, the proposed method take care of spatial texture variation of the images in the form of regional segmentation, which was absent in both the existing methods. The Spearman coeffiecient also shows excellent correlation with the existing metrics [15].

TABLE VI. PPMCC WITH VARIED DISTORTIONS

\begin{tabular}{|l|l|l|l|}
\hline Distortion type & $\begin{array}{l}\text { PSNR vs } \\
\text { Proposed } \\
\text { quality } \\
\text { metrics }\end{array}$ & $\begin{array}{l}\text { PSNR vs } \\
\text { Wang et.al. } \\
\text { quality } \\
\text { metrics }\end{array}$ & $\begin{array}{l}\text { PSNR vs } \\
\text { Shiekh et.al. } \\
\text { quality } \\
\text { metrics }\end{array}$ \\
\hline Gaussian blur & 1 & 1 & -0.1000 \\
\hline $\begin{array}{l}\text { JPEG } \\
\text { Compression }\end{array}$ & 0.9747 & 1 & 0.1429 \\
\hline White noise & 0.7071 & 0.7000 & -1 \\
\hline
\end{tabular}


TABLE VII. SPEARMAN RANK ORDER CORRELATION COEFFIECIENT WITH VARIED DISTORTIONS(PREDICTION MONOTONICITY)

\begin{tabular}{|l|l|l|l|}
\hline Distortion type & $\begin{array}{l}\text { PSNR vs } \\
\text { Proposed } \\
\text { quality metric }\end{array}$ & $\begin{array}{l}\text { PSNR vs } \\
\text { Wang et.al. } \\
\text { quality } \\
\text { metric }\end{array}$ & $\begin{array}{l}\text { PSNR vs } \\
\text { Shiekh et.al. } \\
\text { quality metric }\end{array}$ \\
\hline Gaussian blur & 0.8914 & 0.9146 & 0.0513 \\
\hline $\begin{array}{l}\text { JPEG } \\
\text { compression }\end{array}$ & 0.7650 & 0.4027 & -0.1948 \\
\hline White noise & 0.9299 & 0.6090 & -0.9071 \\
\hline
\end{tabular}

TABLE V. COMPARISON OF PROPOSED QUALITY METRIC WITH OR WITHOUT WAVELET ENTROPY AS A PARAMETER

\begin{tabular}{|c|c|c|c|}
\hline Image name & $\begin{array}{l}\text { H.Sheikh } \\
\text { et.al. }\end{array}$ & $\begin{array}{l}\text { Z.Wang } \\
\text { et.al. }\end{array}$ & $\begin{array}{l}\text { Proposed quality- wavelet } \\
\text { entropy based(linguistic } \\
\text { expression)/MOS/PSNR }\end{array}$ \\
\hline \multicolumn{4}{|c|}{ Gaussian blur } \\
\hline img132 & 78.5510 & 6.6272 & 6 (average) / $8.28 / 32.63$ \\
\hline img162 & 79.7664 & 9.0866 & $\begin{array}{l}9 \text { (good) / } 5.43 / \\
\text { Equivalent to original }\end{array}$ \\
\hline img36 & 74.0736 & 8.0136 & 8.49 (good) / $10 / 34.07$ \\
\hline img42 & 73.4213 & 8.4796 & $8.72($ good) $/ 10 / 34.79$ \\
\hline img61 & 74.4140 & 8.9082 & 9.01 (good) / $10 / 36$ \\
\hline \multicolumn{4}{|l|}{$\begin{array}{l}\text { Jpeg } \\
\text { compressed }\end{array}$} \\
\hline img138 & 79.5797 & -6.1314 & $3.16(\mathrm{bad}) / 1.09 / 30.69$ \\
\hline img1 & 78.9358 & 4.2034 & 6 (average) / $7.57 / 35.09$ \\
\hline img107 & 79.5547 & 7.0294 & $\begin{array}{l}6.01 \text { (average) / } 7.71 / \\
37.35\end{array}$ \\
\hline img154 & 79.6471 & -4.5741 & 6 (average) / $1.09 / 30.76$ \\
\hline img168 & 79.9117 & 9.6770 & 9 (good) / $4.19 / 38.63$ \\
\hline \multicolumn{4}{|l|}{ white noise } \\
\hline img11 & 79.8432 & 9.1115 & 9 (good) / 5.14/39.52 \\
\hline img119 & 80.0207 & 8.6895 & 8.9 (good) / $1.47 / 28.90$ \\
\hline img162 & 79.7664 & 9.0866 & $\begin{array}{l}9(\text { good }) / 6.42 / \text { equivalent } \\
\text { to original }\end{array}$ \\
\hline img28 & 80.0205 & 9.1329 & 9 (good) / $1.95 / 29.72$ \\
\hline img60 & 80.0208 & 6.3326 & 9 (good) / $1 / 28.23$ \\
\hline
\end{tabular}

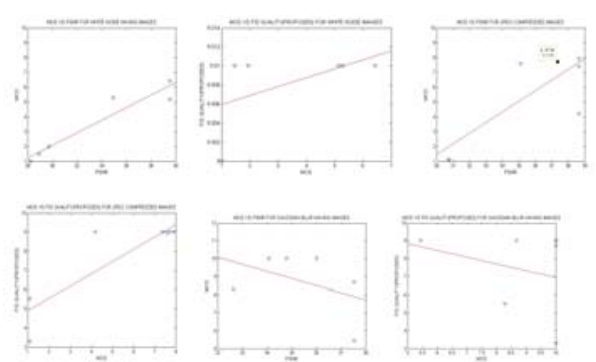

Figure 7. Comparison of MOS vs PSNR and Proposed Quality Metric of different distorted images

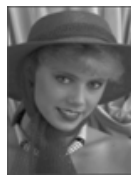

$\operatorname{img} 36$

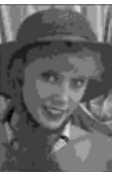

img 138

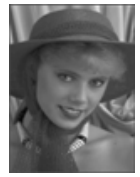

img42

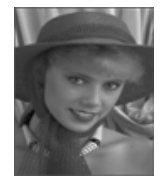

img61

(a)



img 107

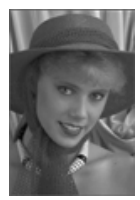

$\operatorname{img} 11$ (b)

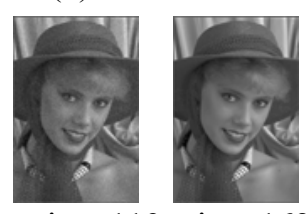

img 119 img 162

(c)
Figure 8. Test Images (a) Gasian Blur (b) Jpeg (c) White Noise

\section{References}

[1] C.T. Lin, C. S. George Lee, Neural Fuzzy Systems, Prentice Hall Ptr, 1993, pp. 140-174.

[2] H. R. Sheikh and A. C. Bovic, "Image information and visual quality". Proc IEEE Int. Conf. Acoust., Speech and Signal Processing, May 2004.

[3] H. R. Sheikh, A. Bovic and L. Cormack, "No-Reference Quality Assesment using Natural Scene Statistics: JPEG 2000," IEEE Transactions on Image Processing., 2005.

[4] Z. Wang, H. Sheikh, A. Bovic, "No Reference Perceptual Quality Assesment of JPEG compressed image," Proceedings of IEEE 2002 International Conferencing on Image Processing, 22-25, 2002.

[5] H. R. Sheikh, Z. Wang, L. Cormack and A. Bovic, "LIVE image quality assessment database 2003," available at http://live.ece.utexas.edu/research/quality.

[6] K. Sayood, Introduction to data compression, pp 267-268, Morgan Kauffman Publishers, 2000.

[7] Z. Wang and A.C. Bovik,"Why is image quality assessment so difficult? "IEEE Int. Conf. Acoust., Speech, and Signal Processing, May 2002

[8] VQEG, Final report from the video quality experts group on the validation of objective models of video quality assessment, http://www.vqeg.org/, Mar. 2000

[9] M. Sonka, V. Hlavac and R. Boyle, Image processing analysis and machine vision, IPT Press, pp. 254-262, 1999.

[10] T. N. Pappas and R. J. Safranek, "Perceptual criteria for image quality evaluation," in Handbook of Image \&Video Proc. (A. Bovik, ed.), Academic Press, 2000.

[11] B. Watson, Ed., Digital Images and Human Vision, Cambridge, MA, MIT Press (1993).

[12] A. B. Watson et al, "Visibility of wavelet quantization noise". IEEE Transactions on image processing, vol. 6, no. 8, pp. 1164-1175, August 1997.

[13] J. C. Dunn, A Fuzzy Relative of the ISODATA Process and Its Use in Detecting Compact Well-Separated Clusters, Journal of Cybernetics 3, 1973: 32-57.

[14] Z. Wang and A.C. Bovik, Modern Image Quality Assessment, Morgan and Claypool publishers, pp.79-102, 2006 
[15] Z. Wang, G. Wu, H.R.Shiekh, E.P. Simoncelli, E.Y. Wang, A.C. Bovik,"Quality aware images", IEEE transactions on image processing, 1680-1689, june 2006

[16] H.Zhen-you, C.xiaoquing, L.Guoming, "Wavelet entropy measure definition and its application for transmission line fault detection and identification;(Part 1: definition and methodology), Powercon 2006, 1-6

[17] Goldberg David E, Genetic Algorithms in Search, Optimization and Machine Learning, Addison-Wesley Pub. Co, 1989.

[18] J.S.Roger-Jang, "ANFIS : Adaptive Network Based Fuzzy Inference System", IEEE Trans. On Systems, Man and Cyberrnetics, vol 23, pp 665-685, May 1993.

\section{Author Biographies}

Indrajit De passed B.Tech in 1997 from Calcutta University and M.Tech in information Technology in 2002 and from Bengal Engineering and Science University, Shibpur. He has three years of work experience as management executive and over eight years as educationist. Presently he is working as Associate Professor in the Department of Information Technology, MCKV Institute of Engineering. His major areas of research interest are fuzzy image processing, image quality, open-source applications.

Jaya Sil Passed B.E. in Electronics and Tele Communication Engg. and M.E. in Computer Science and Engineering in 1984 and 1986 respectively. She was awarded Ph.D. in 1996 and presently professor and Head of the department of Computer Science and Technology of Bengal Engineering and Science University, Shibpur, India. The area of interest of Prof. Sil are Image Processing, Bioinformatics and soft computing techniques 